PROCEEDINGS OF THE

AMERICAN MATHEMATICAL SOCIETY

Volume 137, Number 9, September 2009, Pages 3147-3154

S 0002-9939(09)09905-5

Article electronically published on April 14, 2009

\title{
EFFECTIVE MINIMAL SUBFLOWS OF BERNOULLI FLOWS
}

\author{
ELI GLASNER AND VLADIMIR V. USPENSKIJ
}

(Communicated by Jane M. Hawkins)

\begin{abstract}
We show that every infinite discrete group $G$ has an infinite minimal subflow in its Bernoulli flow $\{0,1\}^{G}$. A countably infinite group $G$ has an effective minimal subflow in $\{0,1\}^{G}$. If $G$ is countable and residually finite, then it has such a subflow which is free. We do not know whether there are groups $G$ with no free subflows in $\{0,1\}^{G}$.
\end{abstract}

\section{INTRODUCTION}

A well known theorem of Ellis asserts that every discrete group admits a free compact flow. This was later extended by Veech to the class of locally compact groups [10]. What Ellis and Veech have actually shown is that the "greatest ambit" of the group $G$ is a free $G$-flow. A $G$-flow is a pair $(X, G)$, where $X$ is a compact Hausdorff space, $G$ is a discrete group, and $G$ acts on $X$ by homeomorphisms. The action is free if no element of $G$ but the identity admits a fixed point. An ambit is a $G$-flow $(X, G)$ with a distinguished point $x_{0} \in X$ whose orbit is dense: $\overline{G x_{0}}=X$. The greatest ambit is the Stone-Čech compactification for a discrete group, and it is the Gelfand space of the Banach algebra $B R U C(G)$ of bounded, right uniformly continuous, complex valued functions on $G$, for a general topological group. For a discrete group $G$, it is known that the enveloping semigroup of the Bernoulli flow on $\Omega=\{0,1\}^{G}$, i.e. the action defined on $\Omega$ by $(g \omega)(h)=\omega\left(g^{-1} h\right)$, coincides with the greatest ambit of $G$. (The enveloping semigroup of a $G$-flow $(X, G)$ is defined as the closure in $X^{X}$ of the set of translations defined by the elements of $G$; for a discussion of the enveloping semigroup including a proof of the above statement, see for example [4, Chapter 1, Section 4].) This implies that, in some sense, the Bernoulli $G$-flow is sufficiently rich to recapture the universal $G$-flow, namely the greatest $G$-ambit. It is thus natural to ask whether for every such $G$, its Bernoulli flow $\left(\{0,1\}^{G}, G\right)$ admits a free subflow. Recently some variants of this question appeared in other contexts as well. In 2 the authors relate some versions of the above problem to combinatorial group theory via tiling, coloring and other geometrical constructions on groups.

Let $e$ denote the identity element of $G$. A flow $(X, G)$ is aperiodic if it does not contain finite orbits. It is minimal if it does not contain proper compact subflows, or, equivalently, the orbit of each point $x \in X$ is dense. The flow $(X, G)$ is effective

Received by the editors June 19, 2007, and, in revised form, December 14, 2007.

2000 Mathematics Subject Classification. Primary 54H20; Secondary 20E99, 37B10.

Key words and phrases. Bernoulli flow, free actions, symbolically-free groups.

The first author is partially supported by BSF grant 2006119 .

(C)2009 American Mathematical Society 
if for every $g \neq e$ in $G$ there is some $x \in X$ with $g x \neq x$. It is strongly effective if there is a point $x_{0} \in X$ such that the map $g \mapsto g x_{0}, g \in G$, is 1-1 from $G$ into $X$. Finally, the flow is free if for every $g \in G \backslash\{e\}$ and every $x \in X$, we have $g x \neq x$.

We introduce the following definitions. Let $G$ be a discrete group. For a positive integer $n \geq 2$ set $\Omega_{n}=\{0,1, \ldots, n-1\}^{G}$ and let $G$ act on $\Omega_{n}$ by left translations:

$$
(g \omega)(h)=\omega\left(g^{-1} h\right), \quad \omega \in \Omega_{n}, g, h \in G .
$$

Then $\left(\Omega_{n}, G\right)$ is the Bernoulli $G$-flow on $n$ symbols. We say that $G$ is symbolically $\{$-aperiodic\}, \{-effective\}, \{-strongly effective\}, $\{$-free $\}$ if for some $n$ the Bernoulli flow $\left(\Omega_{n}, G\right)$ admits an $\{$ infinite minimal $\}$, \{effective $\}$, \{strongly effective $\},\{$ free $\}$ compact subflow, respectively. We denote $\Omega_{2}=\{0,1\}^{G}$ simply by $2^{G}$.

In this paper we show that every infinite group is symbolically-aperiodic, every countable infinite group is symbolically-effective, and every countable infinite residually finite group is symbolically-free. In all these cases we can take $n=2$. More precisely: (1) for every infinite $G$ the Bernoulli flow $2^{G}$ has an infinite minimal subflow (Theorem 2.1); (2) if additionally $G$ is countable (we do not know if this restriction is essential), then there is a minimal effective subflow of $2^{G}$ (Theorem 3.1$)$; (3) if $G$ is countable and residually finite, then there is a minimal subflow of $2^{G}$ on which $G$ acts freely (Theorem 4.2). We provide some further examples of symbolically-free groups and give a combinatorial characterization of this property. We do not know whether there are groups $G$ with no free subflows in $2^{G}$. We would like to thank Vladimir Pestov for the elegant formulation of the combinatorial condition in Section 6 .

What we call symbolically-free or symbolically-aperiodic groups, were called in [2] groups admitting limit aperiodic colorings or limit weakly aperiodic colorings, respectively. We are grateful to Alexander Dranishnikov for making the paper [2] available to us before its publication and for stimulating discussions.

\section{EVERY GROUP IS SYMBOLICALLY-APERIODIC}

2.1. Theorem. For every infinite $G$ the Bernoulli flow $2^{G}$ has an infinite minimal subflow. Thus every infinite group is symbolically-aperiodic.

We need some preliminaries. We have already mentioned that every discrete group $G$ acts freely on the Stone-Cech compactification $S:=\beta G$, which is the greatest ambit of $G$. This is due to Ellis 3 . The proof is by constructing, for every $g$ in $G \backslash\{e\}$, a three-valued function $v: G \rightarrow \mathbb{Z}$ (the integers) such that $v(g h) \neq v(h)$ for every $h \in G$ (an easy exercise), and then extending $v$ to $S$. By Zorn's lemma there is a minimal $G$-flow $M \subset S$. Such an $M$ is called the universal minimal flow, and it is unique up to an isomorphism of $G$-spaces. As the action of $G$ on $S$ is free, $(M, G)$ is a minimal free flow.

A topological space $X$ is extremally disconnected if the closure of every open set $U \subset X$ is clopen, or, equivalently, if disjoint open sets have disjoint closures.

2.2. Lemma (Ellis [3]). For every infinite discrete group $G$ there exists an extremally disconnected minimal compact $G$-space $X$ such that the action of $G$ on $X$ is free.

Proof. The universal minimal compact $G$-space $M$ is a retract of the greatest ambit $\beta G$ (see e.g. 99 or [8, Proof of Lemma 6.1.2]). The Stone-Čech compactification $\beta G$ is extremally disconnected, and it is easy to see that the property of being 
extremally disconnected is preserved by retracts. We have observed that $G$ acts freely on $\beta G$ and hence on $M$.

2.3. Lemma. If $X$ is an infinite extremally disconnected minimal compact $G$-space, then there is a clopen susbset $U \subset X$ such that the $G$-orbit $\{g U: g \in G\}$ of $U$ is infinite.

Proof. Assume, in order to get a contradiction, that every clopen subset of $X$ has a finite $G$-orbit. Let $B$ be the (complete) Boolean algebra of all clopen subsets of $X$. According to our assumption, every $U \in B$ lies in a finite $G$-invariant subalgebra of $B$. It follows that the collection $\mathcal{E}$ of all $G$-invariant finite clopen partitions of $X$ contains "arbitrarily fine" covers: every open cover $\alpha$ of $X$ has a refinement $\beta \in \mathcal{E}$. Consider a sequence $\left\{\gamma_{n}: n \in \mathbb{N}\right\}$ of partitions in $\mathcal{E}$ such that each $\gamma_{n+1}$ is a refinement of $\gamma_{n}$, and each $U \in \gamma_{n}$ is the union of at least three members of $\gamma_{n+1}$. Construct by induction distinct $U_{n}, V_{n} \in \gamma_{n}$ such that $U_{n+1} \cup V_{n+1} \subset V_{n}$. Let $U=\bigcup U_{n}$. We claim that the clopen set $O=\bar{U}$ has an infinite $G$-orbit.

Indeed, $U_{0}$ is the only member of $\gamma_{0}$ contained in $O$, and $U_{n+1}$ is the only member of $\gamma_{n+1}$ disjoint from $U_{n}$ and contained in $O$. It follows that for every $g \in G$ the only disjoint sequence $\left\{W_{n}\right\}$ such that $\bigcup W_{n} \subset g O$ and $W_{n} \in \gamma_{n}$ is the sequence $\left\{g U_{n}\right\}$. Thus for every $n \in \mathbb{N}$ the map $g O \mapsto g U_{n}$ from the $G$-orbit of $O$ onto $\gamma_{n}$ is well-defined (this map is onto because $X$ is minimal). Therefore, the cardinality of the $G$-orbit of $O$ is not less than $\left|\gamma_{n}\right|$. Since $\left|\gamma_{n}\right| \rightarrow \infty$, the orbit of $O$ is infinite.

Proof of Theorem 2.1. According to Lemmas 2.2 and 2.3, there exists a compact minimal $G$-space $X$ and a clopen set $U \subset X$ such that the collection $\{g U: g \in G\}$ is infinite. Let $\chi_{U}: X \rightarrow\{0,1\}$ be the characteristic function of $U$. Consider the $G$-map $\phi: X \rightarrow 2^{G}$ defined by $\phi(x)(g)=\chi_{U}\left(g^{-1} x\right)(x \in X, g \in G)$. Since $X$ is compact and minimal, so is its image under $\phi$. If $x, y \in X$, then $\phi(x)=\phi(y)$ if and only if $x$ and $y$ cannot be separated by a set of the form $g U, g \in G$. It follows that $\phi(X)$ is infinite.

\section{Every Countable group is Symbolically-efFective}

In the proof of the next theorem we will use the following fact. A surjective homomorphism of metric $G$-flows $\pi: X \rightarrow Y$ is called an almost 1-1 extension if the subset $\left\{y \in Y:\left|\pi^{-1}(y)\right|=1\right\}$ is dense and $G_{\delta}$. It is an easy exercise to show that if $\pi$ is an almost 1-1 extension, and if $Y$ is minimal and $X$ is point transitive (i.e. has a point whose orbit is dense), then $X$ is also minimal.

3.1. Theorem. For every discrete infinite countable group $G$ there is a minimal subflow $Y \subset 2^{G}$ and a point $y_{0} \in Y$ such that the map $g \mapsto g y_{0}$ is 1-1. Thus every infinite countable group is symbolically-strongly effective.

Proof. Let $(M, G)$ be a minimal free flow (see the previous section). Fix a point $m_{0} \in M$.

Next consider a metric factor $\pi:(M, G) \rightarrow(X, G)$ such that $\pi \uparrow G m_{0}$ is 1-1; i.e., denoting $x_{0}=\pi\left(m_{0}\right)$, the map $g \mapsto g x_{0}$ from $G$ onto the orbit $G x_{0}$ is 1-1 (this is possible since for a countable $G$ the metric factors separate points on $\beta G$ ). Let $\xi \in X$ be a point which is not in $G x_{0}$. Let $\left\{g_{1}, g_{2}, \ldots\right\}$ be an enumeration of $G \backslash\{e\}$. We construct by induction a sequence of open sets $U_{n} \subset X, n=0,1,2, \ldots$, such that:

(i) for each $n, U_{n+1} \subset U_{n}$, 
(ii) $\bigcap_{n=0}^{\infty} U_{n}=\{\xi\}$,

(iii) for each $n>0, \partial U_{n} \cap G x_{0}=\emptyset$, and

(iv) for every $g_{j} \in G \backslash\{e\}$ there are $h_{j} \in G$ and $n \in \mathbb{N}$ such that $U_{n}$ distinguishes the points $h_{j} x_{0}$ and $h_{j} g_{j} x_{0}$.

Let $d$ be the metric on $X$. We denote by $B_{r}(x)$ the closed ball of radius $r$ centered at $x(x \in X, r>0)$. Let $U_{0}=X$ and let $U_{1}$ be an open set containing $\xi$ which separates $x_{0}$ and $g_{1} x_{0}$ and such that $\partial U_{1} \cap G x_{0}=\emptyset$. Suppose $U_{1} \supset U_{2} \supset \cdots \supset U_{n}$ with $\partial U_{k} \cap G x_{0}=\emptyset$ and that $\operatorname{diam} U_{k}<1 / k, k=2, \ldots, n$, have been constructed. Let $0<\delta<\frac{1}{n+1}$ be such that $B_{\delta}(\xi) \subset U_{n}$ and such that all the points $h_{j} x_{0}$ and $h_{j} g_{j} x_{0}$ for $j \leq n$ are not in $B_{\delta}(\xi)$. By minimality, there is some $g=h_{n+1} \in G$ with $d\left(g x_{0}, \xi\right)<\delta / 2$. Consider the point $z=g g_{n+1} x_{0}$.

A. Case. Suppose $z \notin U_{n}$. Then there is a unique $0 \leq t \leq n-1$ such that $z \in U_{t} \backslash U_{t+1}$. Choose radii $0<2 d\left(g x_{0}, \xi\right)<r_{2}<r_{1}<\delta$ such that $\partial B_{r_{i}}(\xi) \cap G x_{0}=$ $\emptyset, i=1,2$, and set $U_{n+1}=B_{r_{1}}(\xi)$. If $n+1$ and $t$ have the same parity, also set $U_{n+2}=B_{r_{2}}(\xi)$.

B. Case. If $z \in U_{n}$, choose $U_{n+1}$ to be an open set such that $\xi \in U_{n+1} \subset U_{n}$, $\partial U_{n+1} \cap G x_{0}=\emptyset$, with diameter $<\frac{1}{n+1}$, and so that $g x_{0} \in U_{n+1}$ but $z=g g_{n+1} x_{0} \in$ $U_{n} \backslash U_{n+1}$.

This concludes the inductive construction of the sequence $\left\{U_{n}\right\}_{n=0}^{\infty}$. Next define a function $F: X \backslash\{\xi\} \rightarrow\{0,1\}$ by setting $F$ to be 0 and 1 alternately on $U_{n} \backslash U_{n+1}$.

Note that $F$ is continuous at every point of $G x_{0}=\left\{g x_{0}: g \in G\right\}$. Set $y_{0}(g)=$ $f(g)=F\left(g^{-1} x_{0}\right)$; then $f$ is a $\{0,1\}$-valued function on $G$ and the flow it generates in $\{0,1\}^{G}$ under the shift action, say $(Y, G)$, is minimal. In fact the natural joining $Z=X \vee Y$, obtained as the orbit closure in $X \times Y$ of the point $\left(x_{0}, y_{0}\right)$, is an almost 1-1 extension of $X$ and, being point transitive, it is minimal. Therefore $Y$, as a factor of $Z$, is also minimal. Denote by $P_{e}: Y \rightarrow\{0,1\}$ the restriction to $Y$ of the projection of $\{0,1\}^{G}$ onto the $e$-th coordinate. We then have $F\left(g^{-1} x_{0}\right)=$ $f(g)=y_{0}(g)=\left(g^{-1} y_{0}\right)(e)=P_{e}\left(g^{-1} y_{0}\right)$ and, by our construction, for every $g_{j} \in$ $\left\{g_{1}, g_{2}, \ldots\right\}=G \backslash\{e\}$, there is $g \in G$ with

$$
1=\left|F\left(g g_{j} x_{0}\right)-F\left(g x_{0}\right)\right|=\left|f\left(g_{j}^{-1} g^{-1}\right)-f\left(g^{-1}\right)\right|=\left|P_{e}\left(g g_{j} y_{0}\right)-P_{e}\left(g y_{0}\right)\right| .
$$

Thus the map $g \mapsto g y_{0}$, from $G$ into $Y$, is 1-1. This completes the proof.

\section{Residually finite groups are Symbolically-FreE}

We recall that a discrete group $G$ is residually finite when the intersection of all its subgroups of finite index is trivial.

4.1. Lemma. Let $G$ be a discrete infinite countable group. There is then a metrizable zero-dimensional free flow $(X, G)$.

Proof. As we have seen in the proof of Lemma 2.2, the action of $G$ on the universal minimal flow $M$ is free. Let $\left\{f_{g}\right\}_{g \in G \backslash\{e\}}$ be a countable collection of bounded integer valued functions on $G$ with $\inf _{h \in G}\left|f_{g}(g h)-f_{g}(h)\right| \geq 1$ ("Ellis functions"). Let $\mathcal{A}$ be the uniformly closed $G$-invariant subalgebra of $\ell^{\infty}(G)$ containing $\left\{f_{g}\right\}_{g \in G}$ and let $X=|\mathcal{A}|$ be its Gelfand space, with $\pi: \beta G \rightarrow X$ denoting the corresponding canonical map. Then $(X, G)$ is a metrizable flow with $C(X) \cong \mathcal{A}$. For $f \in \mathcal{A}$ let $\hat{f}$ be the corresponding function in $C(X)$. Set $x_{0}=\pi(e)$. 
Given $x \in X$ and $g \in G \backslash\{e\}$, there is a sequence $g_{n} \in G$ with $\lim _{n \rightarrow \infty} g_{n} x_{0}=x$ so that

$$
\hat{f}_{g}(g x)=\lim _{n \rightarrow \infty} \hat{f}_{g}\left(g g_{n} x_{0}\right)=\lim _{n \rightarrow \infty} f_{g}\left(g g_{n}\right)
$$

and

$$
\hat{f}_{g}(x)=\lim _{n \rightarrow \infty} \hat{f}_{g}\left(g_{n} x_{0}\right)=\lim _{n \rightarrow \infty} f_{g}\left(g_{n}\right) .
$$

Thus

$$
\left|\hat{f}_{g}(g x)-\hat{f}_{g}(x)\right|=\lim _{n \rightarrow \infty}\left|f_{g}\left(g g_{n}\right)-f_{g}\left(g_{n}\right)\right| \geq 1 .
$$

In particular $g x \neq x$ and the action $(X, G)$ is free.

Thus we have shown that for every countable infinite $G$ there is a metrizable free $G$-flow $(X, G)$. By [1] (see also [5]) there exists a metrizable zero-dimensional cover $\left(X^{\prime}, G\right) \rightarrow(X, G)$ and we can therefore assume that $X$ is zero-dimensional.

Let us denote by $\mathcal{J}$ the class of groups $G$ which admit an effective metric, zerodimensional, isometric action. If $(X, G)$ is such an action then, since all these properties are preserved in subsystems, we may assume that $(X, G)$ is also minimal. The group Iso $(X)$ of isometries of the zero-dimensional compact metric space $X$ is itself zero-dimensional and compact. To sum up: $G$ is in $\mathcal{J}$ iff it admits a 1-1, metrizable, zero-dimensional, topological group compactification (see e.g. 4]).

A conspicuous subclass of $\mathcal{J}$ is the class of residually finite countable groups. Let $G$ be a residually finite countable group. Let $\mathcal{H}$ be the collection of subgroups $H<G$ with $[G: H]<\infty$. Pick a decreasing sequence $\left\{H_{n}\right\} \subset \mathcal{H}$ such that $\bigcap H_{n}=\{e\}$, and consider the associated inverse limit

$$
X=\lim _{\longleftarrow} G / H_{n}
$$

The corresponding flow $(X, G)$ is topologically a Cantor set, algebraically a compact zero-dimensional topological group, and dynamically an isometric free $G$-action with a bi-invariant metric. Thus $G \in \mathcal{J}$. Note that it was necessary to pass to a countable subsequence of $\mathcal{H}$, since the profinite completion

$$
\stackrel{\lim }{\longleftarrow}_{H \in \mathcal{H}} G / H
$$

of $G$ need not be metrizable.

4.2. Theorem. Let $G$ be an infinite countable discrete group in the class J. Then there is a minimal subflow $Y \subset 2^{G}$ on which $G$ acts freely. Thus every group in J is symbolically-free. In particular this holds for infinite countable residually finite groups.

Proof. Let $X$ be a 1-1, metrizable, zero-dimensional, topological group compactification of $G$ (see Lemma 4.1 above).

Repeat the construction in the proof of Theorem 3.1, with the extra property that $\partial U_{n}=\emptyset$ for every $n$. This can be done as now $X$ is zero dimensional.

Let $\mathcal{C}(F)$ be the set of continuity points of $F$. With this additional condition we have $\mathcal{C}(F)=X \backslash\{\xi\}$ and therefore $\mathcal{C}_{0}(F)=\bigcap\{g \mathcal{C}(F): g \in G\}=X \backslash G \xi$ is a dense $G_{\delta}$ subset of $X$ with a countable complement.

We can now give a full description of the points of $Y$. Set $f(g)=f_{x_{0}}(g)=$ $y_{0}(g)=F\left(g^{-1} x_{0}\right),(g \in G)$. Then for $h \in G$ we have $\left(h y_{0}\right)(g)=y_{0}\left(h^{-1} g\right)=$ $F\left(g^{-1} h x_{0}\right)(g \in G)$. Suppose now that $\lim _{i \rightarrow \infty} h_{i} x_{0}=x$ with $x \in \mathcal{C}_{0}(F)$. We then have

$$
\lim _{i \rightarrow \infty}\left(h_{i} y_{0}\right)(g)=\lim _{i \rightarrow \infty} y_{0}\left(h_{i}^{-1} g\right)=\lim _{i \rightarrow \infty} F\left(g^{-1} h_{i} x_{0}\right)=F\left(g^{-1} x\right) .
$$


Thus in $\{0,1\}^{G}, y_{x}=f_{x}=\lim _{i \rightarrow \infty} h_{i} y_{0}$ exists, with

$$
y_{x}(g)=f_{x}(g)=F\left(g^{-1} x\right)
$$

(so that $y_{0}=y_{x_{0}}$ ). Moreover if $\lim _{i \rightarrow \infty} h_{i} x_{0}=\xi$ and $\lim _{i \rightarrow \infty} h_{i} y_{0}=y$ exists, then for $g \neq e$,

$$
y(g)=\lim _{i \rightarrow \infty}\left(h_{i} y_{0}\right)(g)=\lim _{i \rightarrow \infty} y_{0}\left(h_{i}^{-1} g\right)=\lim _{i \rightarrow \infty} F\left(g^{-1} h_{i} x_{0}\right)=F\left(g^{-1} \xi\right),
$$

and $y(e)$ is either 0 or 1 and accordingly we denote $y=y_{\xi}^{0}$ or $y=y_{\xi}^{1}$. It is now clear that, with the notation $g y_{\xi}^{\epsilon}=y_{g \xi}^{\epsilon}$,

$$
Y=\left\{y_{x}: x \in X_{0}\right\} \cup\left\{y_{g \xi}^{\epsilon}: g \in G, \epsilon=0,1\right\} .
$$

On the dense $G_{\delta}, G$-invariant subset $X_{0} \subset X$ there is a continuous homomorphism $\phi: x \mapsto y_{x}$ from $X_{0}$ into $Y \subset\{0,1\}^{G}$ and (with notation as in the proof of Theorem 3.1)

$$
Z=X \vee Y=\operatorname{cls}\left\{(x, \phi(x)): x \in X_{0}\right\} .
$$

Given $x_{1} \neq x_{2}$ two points in $X_{0}$ we can find a sequence $g_{n} \in G$ such that $\lim _{n \rightarrow \infty} g_{n} x_{1}=\xi$. Since for every $g \in G, d\left(g x_{1}, g x_{2}\right)=d\left(x_{1}, x_{2}\right)$, we can choose some $g \in G$ for which $g x_{1}$ is very close to $\xi$ and

$$
\left|F\left(g x_{1}\right)-F\left(g x_{2}\right)\right|=\left|f_{x_{1}}(g)-f_{x_{2}}(g)\right|=1 .
$$

This shows that the map $\phi: X_{0} \rightarrow Y$ is $1-1$. It is now easy to check that the natural projection map $\pi_{Y}: Z \rightarrow Y$ is 1-1 (an isomorphism) and it follows that the map $\pi: Y \rightarrow X$ defined by $\pi=\pi_{X} \circ \pi_{Y}^{-1}$ (with $\pi_{X}: Z \rightarrow X$ denoting the projection on $X)$ is a continuous homomorphism from $(Y, G)$ onto $(X, G)$. (Explicitly we have $\pi\left(y_{x}\right)=x=\phi^{-1}\left(y_{x}\right)$ for $x \in X_{0}$, and $\pi\left(y_{g \xi}^{\epsilon}\right)=g \xi$ on the complement of $Y_{0}=\phi\left(X_{0}\right)$ in $Y$.)

Thus $(Y, G)$, as an extension of a free action, is itself free and our proof is complete.

\section{Some FuRTher EXAMPLES}

5.1. Theorem. (1) Every Abelian group is symbolically-free.

(2) Every residually finite group is symbolically-free.

(3) Let $S_{\infty}^{0}$ be the group of permutations of $\mathbb{N}$ with finite support. Every infinite subgroup of $S_{\infty}^{0}$ is symbolically-free.

(4) Every torsion free hyperbolic group is symbolically-free.

Proof. 1. Apply Theorem 3.1 and then note that for $G$ Abelian, an effective minimal flow is already free.

2. This is the statement of Theorem 4.2

3. This follows from [6] (see also [8, Section 6.3]) where it is shown that $S_{\infty}$ (the group of all permutations of $\mathbb{N}$ ) admits a minimal action (actually, the universal minimal flow of $S_{\infty}$ as a Polish group) on which the subgroup $S_{\infty}^{0}$ acts freely.

4. This is a result of Dranishnikov and Schroeder, [2].

5.2. Remark. A famous open problem is whether every hyperbolic group is residually finite (see e.g. [7]). An affirmative answer will provide, via Theorem 4.2, a proof that every hyperbolic group is symbolically-free, improving the DranishnikovSchroeder result. 


\section{A COMBINATORIAL CHARACTERIZATION OF SYMBOLICALLY-FREE GROUPS}

In the following theorem we use the pictorial term "a 2-coloring of a set $X$ " to describe a partition of $X$ into two disjoint subsets; each has its own "color". Thus elements $x$ and $y$ of $X$ have "different colors" if they belong to different subsets of the partition.

6.1. Theorem. Let $G$ be an infinite countable group. The following conditions are equivalent.

(1) $G$ acts freely on some subflow of $2^{G}$.

(2) There exists a 2-coloring of $G$ with the following property. For every $g \neq e$, there is a finite set $A=A(g)$ such that for every $h \in G$ there is an $a \in$ $A \cap g^{-1} A$ such that ha and hga have different colors.

Proof. Suppose first that $Y \subset \Omega_{2}=\{0,1\}^{G}$ is a free subflow. Let $\omega$ be any point in $Y$. We consider $\omega$ as a coloring of $G$ and will show that it has the property described in (2). If this is not the case, then there is some $g \in G \backslash\{e\}$ such that for every finite set $A \subset G$ there is some $h=h_{A} \in G$ with the property that $\omega(h g a)=\omega(h a)$ for every $a \in A \cap g^{-1} A$. Let $A_{n}$ be an increasing sequence of finite subsets of $G$ with $G=\bigcup_{n=1}^{\infty} A_{n}$. For each $n$ let $h_{n}=h_{A_{n}}$ be the corresponding element of $G$ such that

$$
h_{n}^{-1} \omega(g a)=\omega\left(h_{n} g a\right)=\omega\left(h_{n} a\right)=h_{n}^{-1} \omega(a) \quad \text { for every } a \in A_{n} \cap g^{-1} A_{n} .
$$

Clearly also $G=\bigcup_{n=1}^{\infty}\left(A_{n} \cap g^{-1} A_{n}\right)$, and taking a convergent subsequence $h_{n_{i}}^{-1} \omega \rightarrow$ $\xi \in Y$, we have

$$
\xi(g a)=\xi(a) \text { for every } a \in G .
$$

Thus $g \xi=\xi$, contradicting our assumption that the flow $(Y, G)$ is free.

Conversely, assume now that condition (2) is satisfied. Let $\omega: G \rightarrow\{0,1\}$ be the coloring whose existence is ensured by this condition and consider its orbit closure $Y=\operatorname{cls}(G \omega)$ in $\Omega_{2}$. Let $\xi$ be any point in $Y$ and let $g$ be an element of $G \backslash\{e\}$. There exists a sequence $h_{n} \in G$ with $\xi=\lim _{n \rightarrow \infty} h_{n}^{-1} \omega$. This means that for any finite $A \subset G$, eventually

$$
h_{n}^{-1} \omega(a)=\omega\left(h_{n} a\right)=\xi(a) \text { for every } a \in A .
$$

In particular this holds for the finite set $A=A\left(g^{-1}\right)$ given in condition (2) and we fix some $h=h_{n}$ for which equation (11) holds with respect to this $A$. By condition (2) then, there exists some $a \in A \cap g^{-1} A$ with $\omega(h a) \neq \omega(h g a)$. But then

$$
\xi(a)=h^{-1} \omega(a)=\omega(h a) \neq \omega(h g a)=h^{-1} \omega(g a)=\xi(g a)=g^{-1} \xi(a) .
$$

Thus $g^{-1} \xi \neq \xi$ and we have shown that $(Y, G)$ is a free flow.

Call the condition (2) in Theorem 6.1 property $P$. With this terminology, each of the groups listed in Theorem 5.1 has property $P$. The main question now is whether there is any countably infinite group which does not satisfy property $P$. Stated explicitly we have the following.

6.2. Problem. Is there a countable infinite group $G$ with the following coloring property?

For every 2-coloring of $G$, there exists $g \in G \backslash\{e\}$ such that for every finite set $A \subset G$, there exists an $h \in G$ for which the pair $h a$ and $h g a$ have the same color for every $a \in A \cap g^{-1} A$. 
6.3. Remark. After this paper was accepted for publication, we learned that our main question (formulated as the coloring problem 6.2) had recently received a negative answer by Su Gao, Steve Jackson, and Brandon Seward. This paper, entitled "A coloring property for countable groups", is available on Gao's homepage

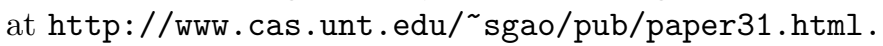

\section{REFERENCES}

[1] M. Boyle, D. Fiebig and U. Fiebig, Residual entropy, conditional entropy and subshift covers, Forum Math. 14 (2002), 713-757. MR1924775 (2003g:37024)

[2] A. Dranishnikov and V. Schroeder, Aperiodic colorings and tilings of Coxeter groups, Groups Geom. Dyn. 1 (2007), 311-328. MR2314048

[3] R. Ellis, Universal minimal sets, Proc. Amer. Math. Soc. 11 (1960), 540-543. MR0117716 $(22: 8491)$

[4] E. Glasner, Ergodic Theory via Joinings, Math. Surveys and Monographs, vol. 101, Amer. Math. Soc., Providence, RI, 2003. MR.1958753 (2004c:37011)

[5] E. Glasner and B. Weiss, Quasi-factors of zero-entropy systems, J. Amer. Math. Soc. 8 (1995), 665-686. MR1270579 (95i:54048)

[6] E. Glasner and B. Weiss, Minimal actions of the group $\mathbb{S}(\mathbb{Z})$ of permutations of the integers, Geom. Funct. Anal. 12 (2002), 964-988. MR1937832 (2003h:37009)

[7] I. Kapovich and D. T. Wise, The equivalence of some residual properties of word-hyperbolic groups, J. Algebra 223 (2000), 562-583. MR1735163 (2001f:20086)

[8] V. Pestov, Dynamics of Infinite-Dimensional Groups. The Ramsey-Dvoretzky-Milman Phenomenon, University Lecture Series, vol. 40, Amer. Math. Soc., Providence, RI, 2006. MR2277969 (2008c:37009)

[9] V. Uspenskij, On universal minimal compact G-spaces, Topology Proceedings 25 (2000), 301-308. MR1875600 (2002j:22024)

[10] W. A. Veech, Topological dynamics, Bull. Amer. Math. Soc. 83 (1977), 775-830. MR0467705 $(57: 7558)$

Department of Mathematics, Tel-Aviv University, Tel Aviv, Israel

E-mail address: glasner@math.tau.ac.il

URL: http://www.math.tau.ac.il/ glasner

Department of Mathematics, 321 Morton Hall, Ohio University, Athens, Ohio 45701

E-mail address: uspensk@math.ohiou.edu 